\title{
Étude de l'amélioration des conditions de navigabilité du Niger dans la République du Mali
}

\section{Study of methods of improving navigability conditions on the Niger, in the Republic of Mali}

\author{
PAR MM. CHABERT ET REMILLIEUX
}

INGÉNIEURS AU LABORATOIRE NATIONAL D'HYDRAULIQUE

\begin{abstract}
La navigation sur le Niger en aval de Bamako, particulièrement entre Koulikoro et Ségou, est qênée en étiage par la présence de senils sableux.

Un modèle réduit d'un tronçon caractéristique du Niger a été construit au Laboratoire National d'Hydraulique, tandis que celui-ci dirigeait les mesures in situ et mettait en place des panneaux déviateurs: «panneaux de fond》 fixés sur les fonds sableux des seuils et constituant un système de correction définitif, et systèmes mobiles de «panneaux de surface» agissant $\dot{a}$ la manière d'une drague.
\end{abstract}

\begin{abstract}
Sand bars are a considerable hindrance to navigation in the Niger below Bamako during low water periods, especially between Koulikoro and Segou.

A scale model of a typical section of the Niger was built at the "Laboratoire National d'Hydraulique", which also supervised on-site measurements and installed "deflecting panels" in the river itself. The latter were provided in two forms: 1) "Bottom panel", which were fixed to the sandy bed on the bars and 2) Mobile "surface panels" acting much in the same way as a dredge.
\end{abstract}

Les conditions actuelles de navigabilité du Niger font que toutes les possibilités offertes par cette voie d'eau ne sont pas utilisées sur cet axe naturel de transport en République du Mali.

Le bief Koulikoro-Ségou a une situation économique prédominante (fig. 1) ; en effet, Koulikoro, situé à environ $60 \mathrm{~km}$ en aval de Bamako, est le terminus du chemin de fer Dakar-Niger, et Ségou est le siège de l'Office du Niger qui a aménagé un périmètre de 50000 ha dont l'irrigation est contrôlée par le barrage de MarkalaSansanding. Ce bief de $180 \mathrm{~km}$, qui ne représente qu'une faible longueur des $1400 \mathrm{~km} \mathrm{na-}$ vigables en aval de Koulikoro, assure cependant $45 \%$ du trafic total descendant et $95 \%$ du trafic total montant, soit un tonnage moyen annuel de 60000 à 65000 tonnes.

Mais près de cinq mois par an en moyenne, de la mi-janvier à la mi-juin, la navigation devient progressivement très difficile, puis doit s'arrêter complètement, en raison de la baisse du niveau des eaux du Niger et de l'extrême irrégularité des fonds de son lit sableux. En particulier, l'exportation des produits agricoles de l'Office du Niger est affectée par cette interruption. 


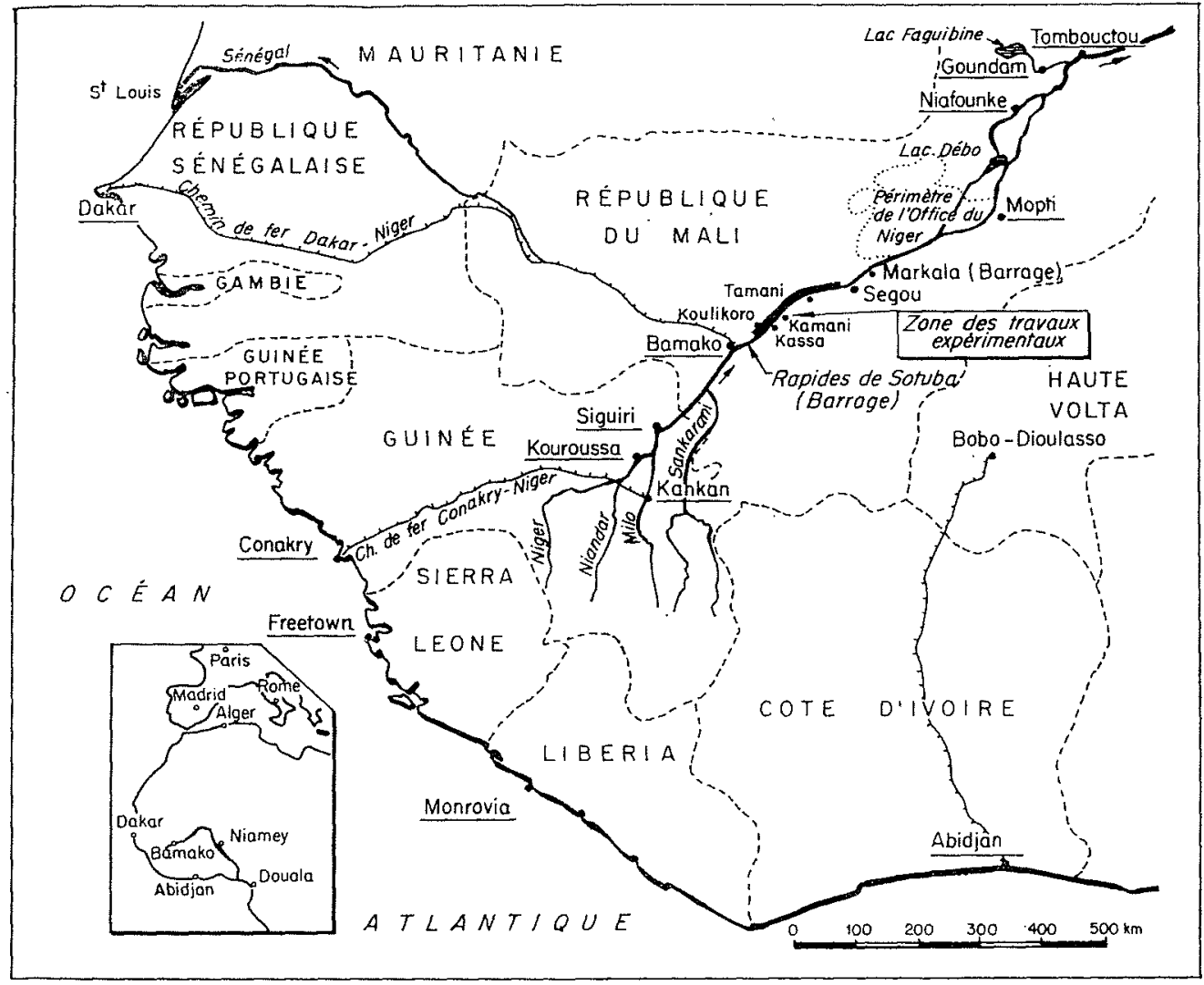

Fig. 1

Plan de situation.

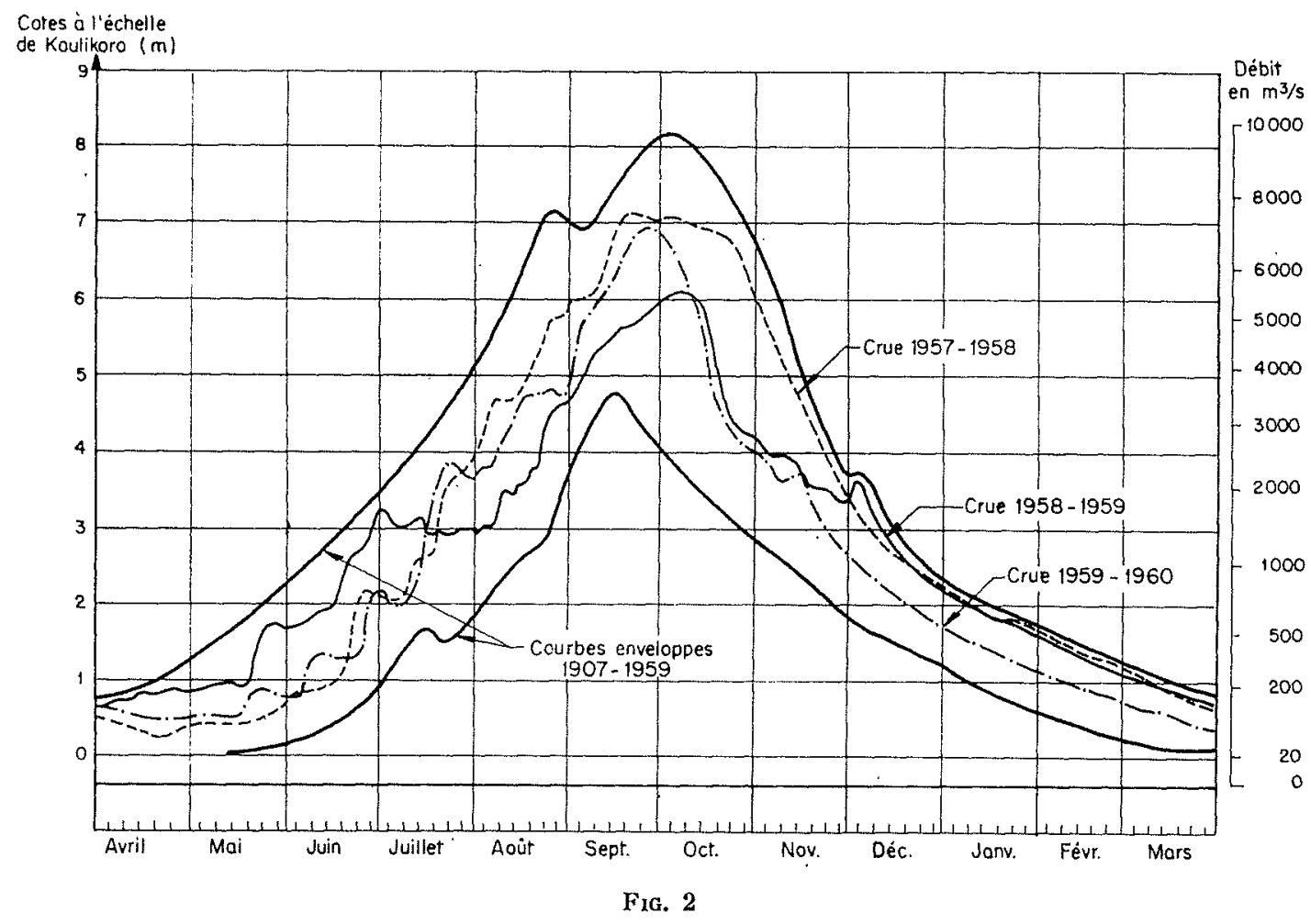

Courbes de crue du Niger. 
FiG. 3

Vue aérienne d'un seuil lors de l'étiage.

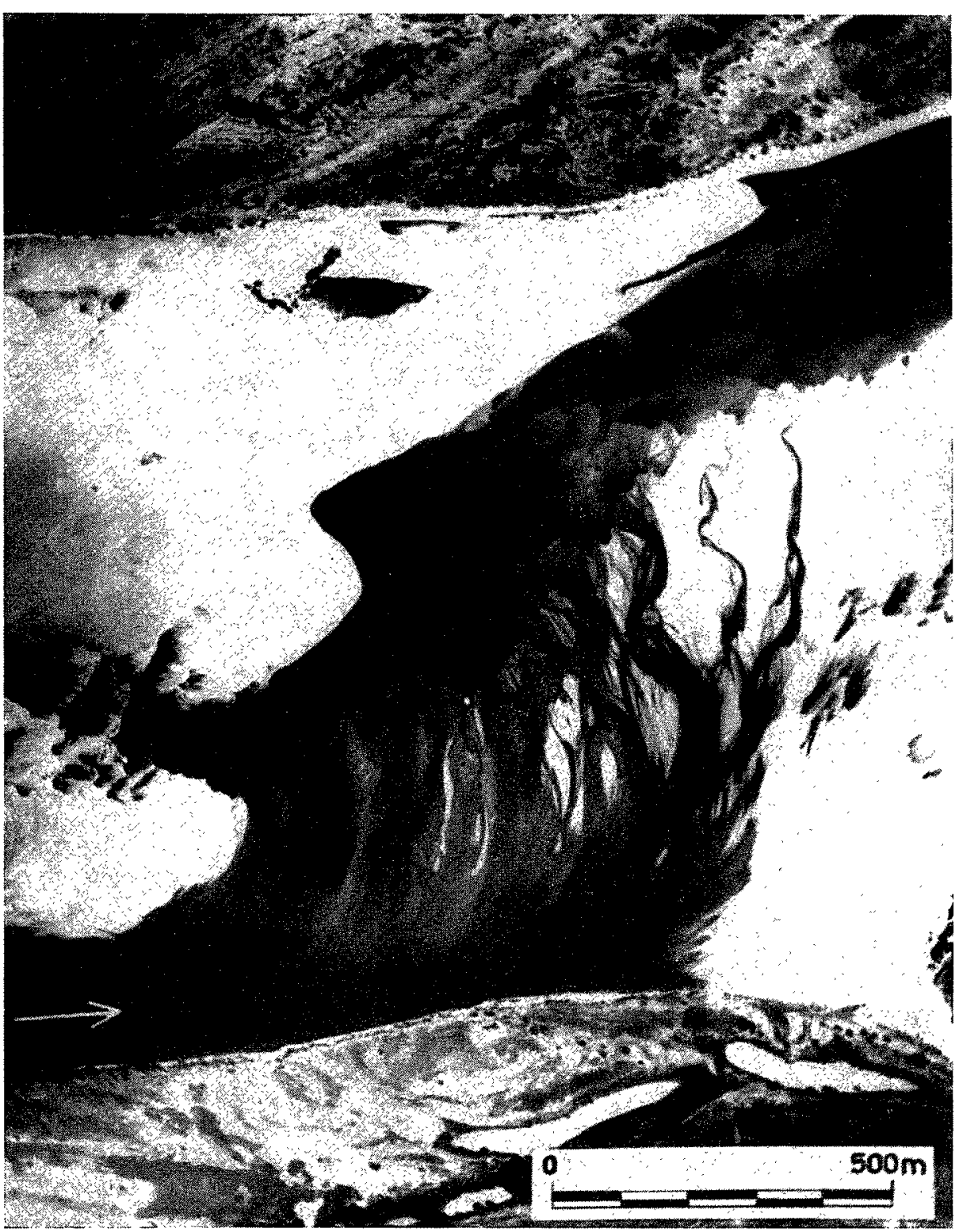

\section{PRINCIPALES DONNEESS RELATIVES AU NIGER}

\section{Données hydrologiques.}

Le régime du Niger, dont le bassin versant à Koulikoro s'étend sur $120000 \mathrm{~km}^{2}$ principalement situés en Guinée, a un régime qui se caractérise par une crue unique simple (fig. 2).

Les lectures journalières effectuées depuis 1907 à l'échelle de Koulikoro, dont le zéro fixe la cole de l'étiage conventionnel, permettent de connaître avec précision les caractéristiques hydrologiques du fleuve sur le bief Koulikoro-Ségou.
La crue commence en juin, atteint son maximum en septembre-octobre (4500 à $10000 \mathrm{~m}^{3} / \mathrm{s}$ ) et décroît progressivement jusqu'à l'étiage en mars-avril-mai $\left(20\right.$ à $\left.200 \mathrm{~m}^{3} / \mathrm{s}\right)$.

\section{Données topographiques.}

Entre Koulikoro et Ségou, le Niger coule avec une faible pente, de l'ordre de $7 \mathrm{~cm} / \mathrm{km}$, sur un lit formé de sables ayant un diamètre moyen de 
$1 \mathrm{~mm}$. La largeur de ce lit est en moyenne de $1200 \mathrm{~m}$, mais, en période de hautes eaux, le cliamp d'inondation peut atteindre 5 à $6 \mathrm{~km}$. Par contre, aux moyennes et basses eaux, les bancs de sable se découvrent et le lit mineur' se réduit à une largeur de 600 à $200 \mathrm{~m}$.

Le lit mineur est formé d'une succession de mouilles, où la navigation est aisée, et de seuils formant obstacle sur des longueurs pouvant atteindre $800 \mathrm{~m}$. Il y a étalement du débit d'étiage sur ces seuils et le tirant d'eau peut y tomber à moins de $20 \mathrm{~cm}$ (fig. 3 ).

\section{Caractéristiques de la navigation.}

Ce bief est surtout exploité par la Société Fermière des Messageries Africaines dont le tonnage kilométrique annuel est de 3.0 à 35 millions.

Les chalands, d'un tonnage de 50 à 150 tonnes, calent de 0,80 à $1,60 \mathrm{~m}$. Ils sont tractés par des remorqueurs de 100 ou $200 \mathrm{ch}$ Diesel qui ne limitent pas le tirant d'eau à pleine charge.
On pratique alors différents types de navigation : lourde, semi-lourde et légère, suivant que le tirant d'eau disponible est de 1,50 m, 1,20 $\mathrm{m}$ ou $0,80 \mathrm{~m}$; ceci correspond à des cotes à l'échelle de Koulikoro respectivement de $1,95 \mathrm{~m}, 1,75 \mathrm{~m}$ et $1,35 \mathrm{~m}$, compte tenu de la cote relative des seuils les plus mauvais.

En moyenne, la navigation lourde est interrompue de la mi-décembre à début juillet, la navigation semi-lourde de la mi-janvier à la mi-juin, et la navigation légère de début février à début juin. En fait, la navigation se poursuit encore avec talonnage de fond et échouages, voire même transbordement, en sorte que le trajet KoulikoroSégou, qui dure normalement un à deux jours, peut dépasser quinze jours.

Cette flotte commence à être adaptée au poussage; des remorqueurs ont été transformés en pousseurs et des barges ont été construites. Le poussage, qui remplacera la navigation lourde et semi-lourde, ne nécessitera plus que $1,30 \mathrm{~m}$ de tirant d'eau. Le poussage allégé permettra encore la navigation avec des tirants d'eau de 0,55 à $0,60 \mathrm{~m}$.

\section{HISTORIQUE DES TENTATIVES D'AMELIORATION DE LA NAVIGABILITE}

Les études hydrographicues ont commencé en 1904.

En 1936, à partir de février, on procéda à des dragages étroits effectués à la main sur une quarantaine de seuils, travail qui nécessita l'équivalent de dix mille journées. En avril, il ne restait rien des travaux effectués sur la moitié des seuils traités, et si un gain de quelques dizaines de centimètres a pu être obtenu sur les autres seuils, c'est au prix d'un entretien permanent.

En 1938, après un nouveau levé hydrographique, un projet d'aménagement à courant libre fut établi en profitant des expériences faites sur le Rhône à la fin du $x_{1 x^{\circ}}$ siècle; il prévoyait la mise en place de $400000 \mathrm{~m}^{3}$ d'enrochements, d'ailleurs fort difficiles à trouver, répartis sur vingt-cinq seuils, sous forme d'épis, digues, tenons et traverses (coût réévalué de plus de 20 millions de NF).
$16000 \mathrm{~m}^{3}$ d'enrochements, formant près de $6 \mathrm{~km}$ d'ouvrage, furent placés sur un seuil d'essai. Mais ces travaux ne furent d'aucune utilité et l'équipement des autres seuils ne fut jamais réalisé. De nombreux enrochements ont même dû être enlevés à la main, car plusieurs épis sont en travers du chenal actuel, qui n'est plus celui que l'on avait cherché à stabiliser.

En 1947, le projet d'un barrage-réservoir en Guinée fut étudié (coût de 60 à 160 millions de $\mathrm{NF}$ ), mais il est rapidement apparu qu'il était très onéreux d'améliorer la navigabilité uniquement par régularisation des débits d'étiage.

En 1957, après un nouveau levé hydrographique, l'étude de l'amélioration des conditions de navigabilité du Niger entre Koulikoro et Ségou fut confiée au Laboratoire National d'Hydraulique. 


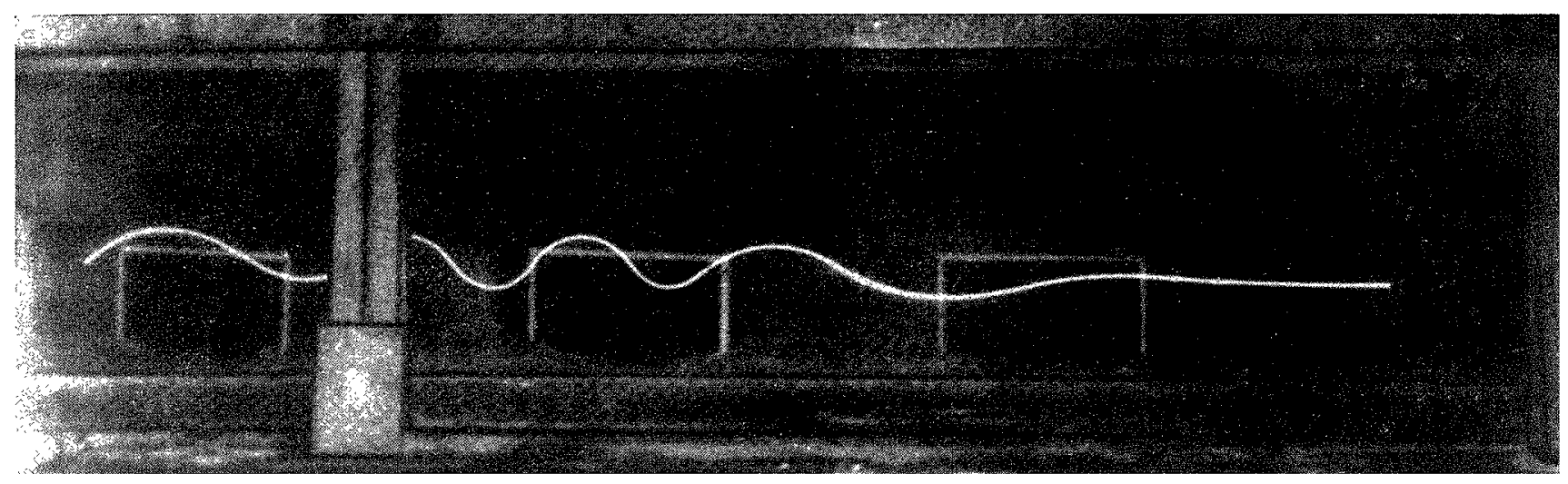

FIG. 4

Visualisation du mouvement hélicoüdal.

\section{PRINCIPE DE LA CORRECTION PROPOSEE}

Dès la fin $d u x_{I} x^{*}$ siècle, Fargue a montré que la forme stable du lit d'une rivière était un chenal sinueux présentant une succession de mouilles et de seuils. Les observations et les études effectuées ensuite ont, en particulier, mis en évidence l'influence de l'écoulement hélicoïdal dans la formation des mouilles.

Ce mouvement hélicoïdal, qui se produit naturellement dans les courbures, peut être réalisé artificiellement pour créer des affouillements locaux au moment et à l'endroit voulu.

Potapov, en particulier, étudia, à partir de 1932, la création du mouvement hélicoïdal en canal au moyen de panneaux déviateurs placés près du fond ou près de la surface. Parallèlement Prostov, Lossievsky, Katchatrian utilisèrenl ce principe pour corriger des rivières ou protéger de l'ensablement les prises d'eau.

A partir de ces études et résultats, le Laboratoire National d'Hydraulique a effectué de nombreux essais de mise au point, tant sur modèle de rivière qu'en canal expérimental, pour des panneaux de fond et des panneaux de surface.

La figure 4 visualise le mouvement hélicoïdal obtenu en canal expérimental.

\section{Panneaux de fond.}

Le dispositif de correction par panneaux de fond se présente comme une série de seuils noyés disposés suivant un angle très aigu par rapport au courant.

La figure 5 montre comment se crée le chenal en canal expérimental; une partie des malériaux est ramenée par le mouvement hélicoïdal contre les panneaux et les protège ainsi contre l'afrouillement.

Les panneaux de fond constituent un système. de correction définitif au même titre que les digues ou épis; leur implantation doit être elTectuéc en fonction des conditions hydrauliques el topographiques locales.

\section{Panneaux de surface.}

Un mouvement hélicoüdal double peut être crée par des panneaux de surface faiblement immergés et judicieusement orientés; la convergence des courants de surface entraîne une ćrosion axiale du lit par la divergence des courants de fond.

La figure 6 montre comment un sysleme de panneaux de surface, supporté par quatre bras, crée le chenal en canal expérimental.

Les panneaux de surface ont une action comparable à celle d'une drague, mais ils présentent de plus l'avantage de fonctionner d'une facon continue sous l'action de l'écoulement, de ne pas poser le problème du rejet des sables dragués et de ne pas gêner le passage de la navigation (en effet, du fait de leur longueur d'action, ils peuvent être placés légèrement en amont du seuil à traiter).

Ces panneaux peuvent être adaptés pour eviter l'ensablement des prises d'eau; le Laboratoire $\mathrm{Na}$ tional d'Hydraulique a en particulier étudié par ce procédé la protection de la prise d'eau alimentant tout le périmètre agricole de l'office du Niger. 

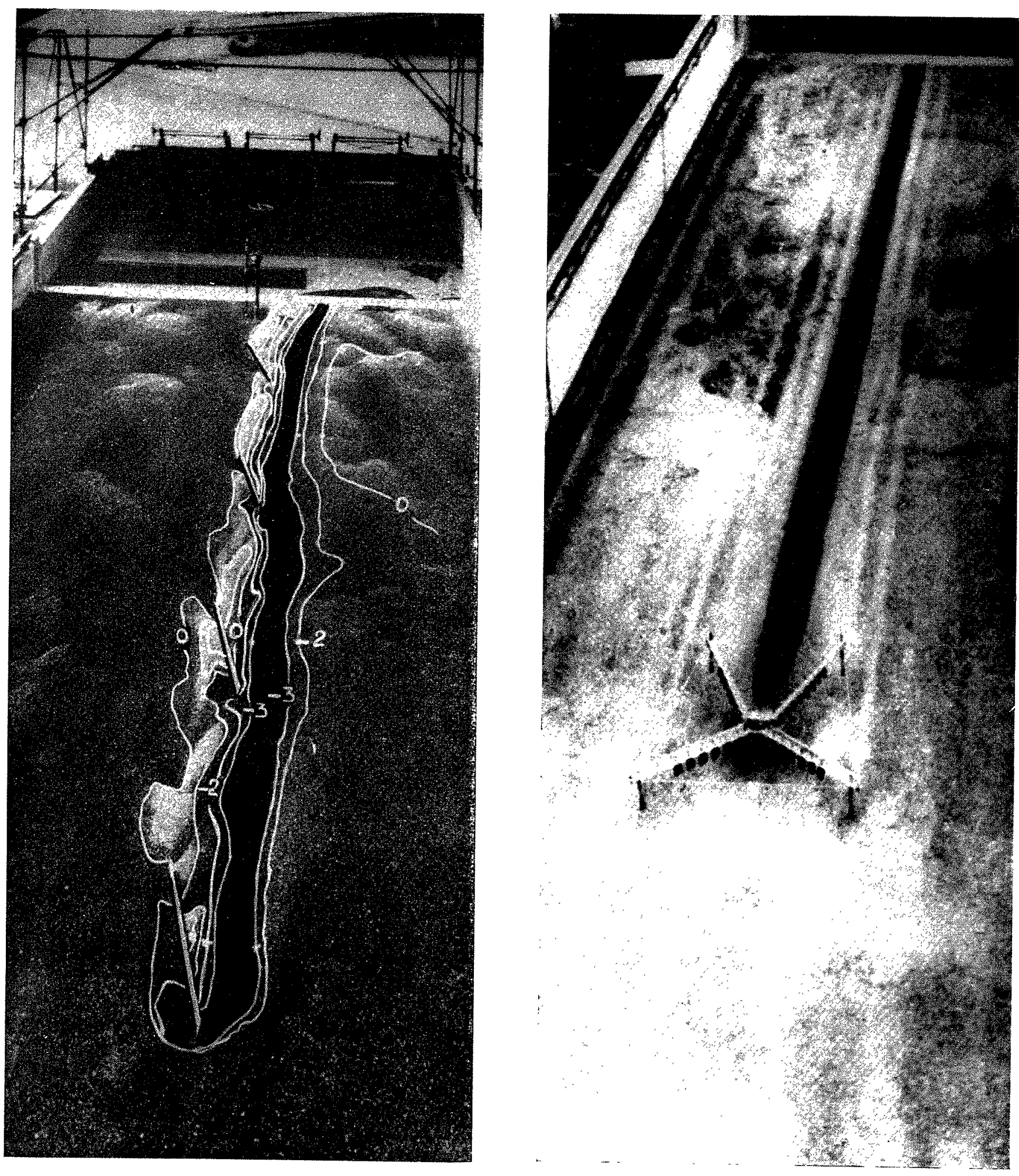

Fia. 5

FIG. 6

Essais de panneaux de fond en canal.

Fssais de panneaux de surface en canal. 


\section{MESURES EFFECTUÉES SUR PLACE ET SUR MODÉLE}

L'adaptation de cette méthode au bief Koulikoro-Ségou a été réalisée à la suite de deux campagnes de mesures et de travaux expérimentaux effectués sur place sous la direction du Laboratoire National d'Hydraulique, et d'une étude sur modèle, effectuée au Laboratoire, d'un tronçon caractéristique du bief.

Les mesures et travaux furent concentrés principalement sur les deux tronçons caractéristicjues de Kamani et de Kassa.

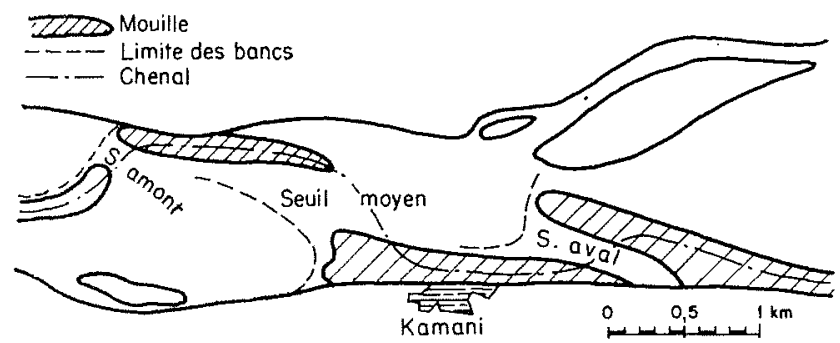

FIG. 7

Croquis de la région de Kamani.

La figure 7 donne le croquis de la région de Kamani ( $7 \mathrm{~km}$ environ), avec ses trois seuils dont le principal est le seuil central (ou seuil moyen) qui correspond à un changement de rive du chenal.

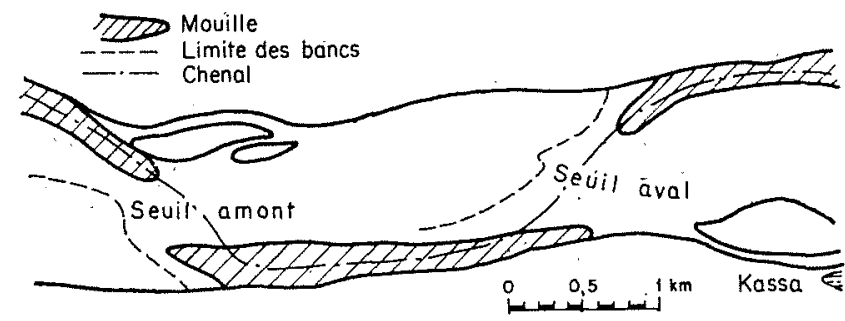

FIG. 8

Croquis de la région de Kassa.

La figure 8 correspond à la région de Kassa, où se succèdent deux grands seuils avec changement de rive du chenal.

\section{Mesures effectuées sur place d'octobre 1957 à novembre 1958.}

Elles étaient principalement destinées à permettre le réglage du modèle et à préparer l'im- plantation d'ouvrages dans les tronçons expérimentaux; elles comprenaient essentiellement:

- des levés hydrographiques et des mesures de la ligne d'eau à différentes époques de la crue pour suive l'évolution des fonds et en particulier l'avancement des grands bancs de sable;

-. des analyses granulométriques des sables;

- Ia détermination de l'évolution du champ des vitesses superficielles en cours de décrue et des vitesses au voisinage des fonds sableux;

-.- la détermination directe du mouvement des sables.

On a utilisé pour cette dernière mesure des tracenrs radioactifs : une petite quantité de verre contenant du Ta 182, ayant la densité ef. la granulométrie du sable naturel, est activée dans une pile atomique et déposée, au moyen d'un appareil spécial, sur les fonds sableux.

L'évolution des zones radioactives peut être suivie suffisamment longtemps (période 111 jours) pour en déduire l'allure des mouvements des fonds sableux.

Neuf dépôts ont été efrectués sur des zones caractéristiques en étiage et en cours de décrue. La figure 9 (dépôts A et B) montre que, dans le chenal en étiage, les trajectoires sont presque filiformes et que l'avancement moyen est de $7 \mathrm{~m}$ par jour. Sur un seuil à la décrue, il y a au contraire une forte dispersion latérale (dépôts III et IV) et une vitesse d'avancement très variable d'un point à l'autre (il $y$ a pratiquement immobilité au point V).

\section{Le modèle.}

Les essais, effectués au Laboratoire sur le tronçon caractéristique de Kamani, avaient pour but de définir :

- l'implantation des ouvrages pour obtenir leur efficacité optimale et le maintien du chenal à Iong terme;

- le meilleur moment de mise en place des ouvrages;

- l'effet sur le chenal ainsi cré d'une régularisation éventuelle du débit d'étiage par suite de la construction d'un barrage-réservoir;

- - les règles d'implantalion des ouvrages pour 


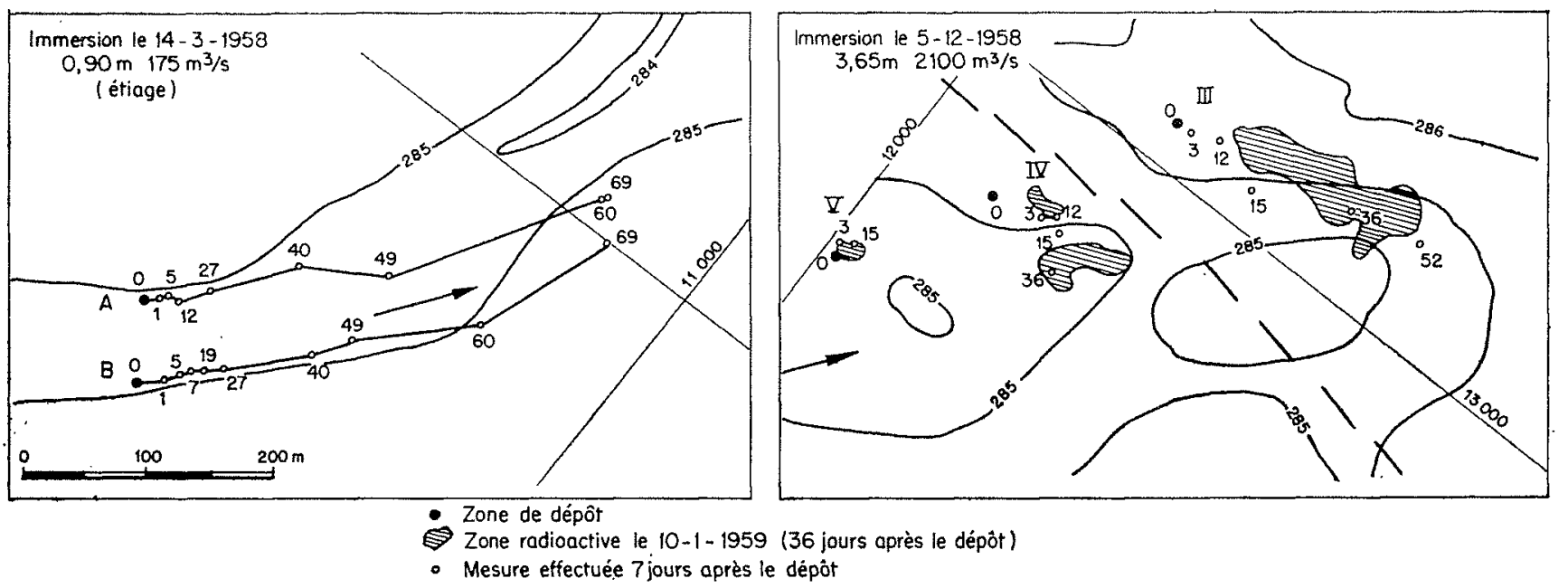

FIG. 9

Traceurs radionctifs (nature).

les autres seuils à aménager entre Koulikoro et Ségou à partir de ces résultats.

\section{Caractéristiques du modèle.}

L'échelle en plan, compatible avec les conditions aux limites imposées, est le $1 / 150^{\circ}$. Les autres échelles et les caractéristiques du matériau de fond ont été choisies pour satisfaire aux conditions suivantes :

- condition de turbulence et de début de charriage en notant que l'écoulement naturel n'est déjà pas en turbulence complète sur le fond (il faut en effet éviter les phénomènes de rides parasites sur le modèle);

- condition de rugosité, en adoptant une formule de perte de charge logarithmique en raison des valeurs très différentes du rapport de la profondeur à la dimension des aspérités, dans la nature et sur le modèle;

- condition de charriage (cette dernière condition donne l'échelle des temps de charriage).

Les caractéristiques finalement adoptées sont les suivantes :

- échelle en plan 1/150

-. échelle en hauteur $1 / 40$

distorsion 3,75

- échelle des pentes 18

basculement $1,11^{--3}$

- échelle des vilesses $1 / 5$

échelle des débits $1 / 30000$
Le matériau de fond est du noyau d'abricot concassé; densité 1,30 , diamètre moyen $2,6 \mathrm{~mm}$. échelle des temps de charriage $1 / 720$, soit deux minutes pour représenter un jour.

\section{Etalonnage du modèle.}

Il a pu être très complet, grâce au grand nombre de mesures effectuées dans la nature : lignes d'eau, levés hydrographiques, vitesses, etc.

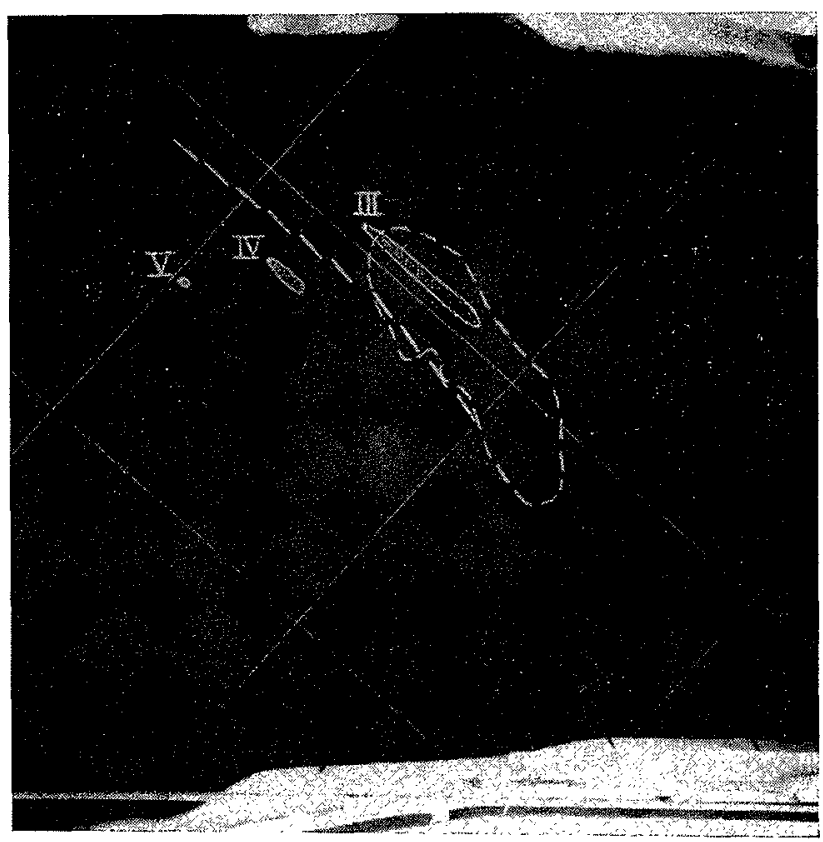

FIG. 10

Traceurs radioactifs (modèle). 
La figure 10 montre, par exemple, l'évolution de traceurs modèle. La zone de dispersion de traceurs colorés pour un temps correspondant à 36 jours nature (trait continu) est en bonne concordance avec la figure 9 . De plus, une petite quantité de matériau du modèle activé et placé au point homologue du point III a permis de prolonger l'expérience pendant 96 jours nature (trait tireté).

Les panneaux implantés, avant toute étude sur modèle, lors de la première campagne, ont été mis en place sur le modèle et leur effet sur les seuils a ainsi été contrôlé (fig. 11). Leur action a ensuite

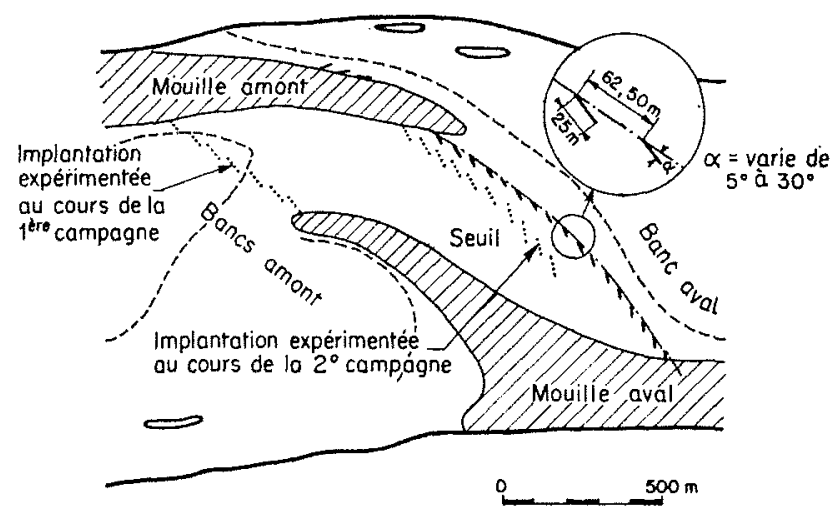

FIG. 11

Schéma d'implantation des panneaux de fond (région de Kamani).

été prolongée en réalisant de nouvelles crues sur le modèle; certains panneaux se sont ensablés, conformément à ce qui a été vérifié ultérieurement dans la nature après la crue de 1958 .

\section{Résultats principaux des essais.}

Les essais ont permis de dégager des régles d'implantation des panneaux de fond :

- il faut implanter les panneaux de fond à partir de l'aval de la mouille amont vers la mouille aval, pour que l'ensemble des pan- neaux soit le plus loin possible de la dune amont, sinon les panneaux risquent de s'ensabler lors des premières crues et de n'avoir ensuite plus aucun effet;

- la direction de l'écoulement devenant de plus en plus oblique entre les deux mouilles au fur et à mesure de la décrue, l'inclinaison des panneaux est déterminée pour que l'angle d'attaque du courant sur chacun d'eux corresponde à leur meilleure efficacité pendant la période de la décrue où le chenal doit se créer;

- il y a intérêt à augmenter le nombre des panneaux de fond et à diminuer leur longueur unitaire. L'espacement optimal entre les têtes de deux panneaux est voisin de deux fois et demie leur longueur;

- enfin quelques panneaux sont nécessaires pour fixer les conditions aux limites amont en évitant le déplacement de la mouille amont vers l'aval par remblaiement de son extrémité.

Une première implantation a été mise au point très rapidement sur le modèle (panneaux de $50 \mathrm{~m}$ ) et immédiatement réalisée sur place au cours de la seconde campagne de travaux expćrimentaux (fig. 11).

Les essais qui ont ensuite été poursuivis ont permis de mettre au point l'implantation définitive (panneaux de $25 \mathrm{~m}$ ). Sur la figure 12 , on voit l'implantation des panneaux de fond mise au point pour les trois seuils représentés sur le modèle.

L'expérience acquise sur le modèle permet de fixer l'implantation des panneaux pour chaque seuil, compte tenu du levé hydrographique et de la détermination du champ des vitesses, qui seront effectués immédiatement avant les travaux.

D'autre part, la poursuite des essais en canal expérimental a permis d'adapter un système de panneaux de surface aux conditions hydrauliques du Niger.

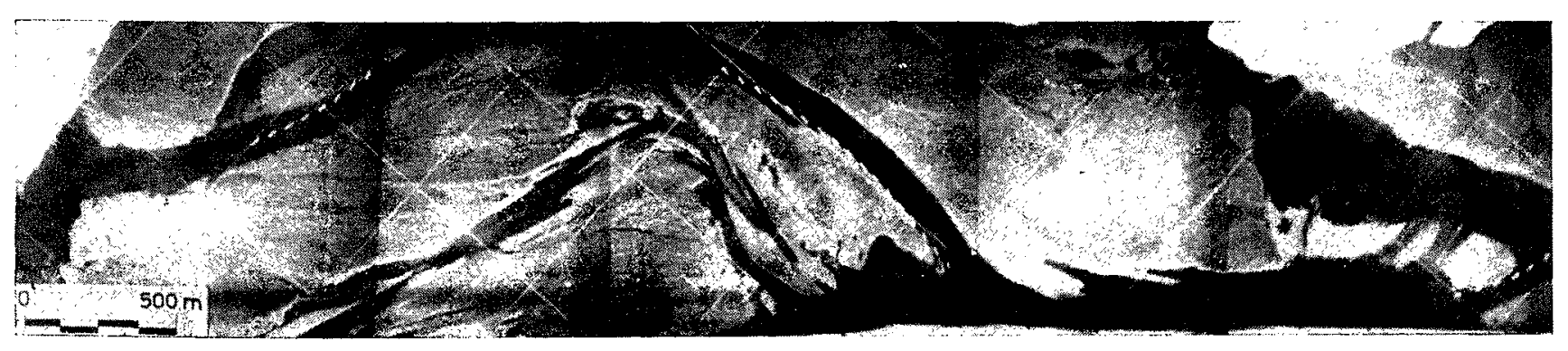

Fig. 12

Vue générale du modèle. 


\section{RÉSULTATS DES TRAVAUX EXPÊRIMENTAUX EFFECTUÉS DANS LA NATURE}

Les travaux expérimentaux ont été effectués principalement dans les tronçons caractéristiques du Niger de Kamani et de Ǩassa, sous la direcLion du Laboratoire National d'Hydraulique, en deux campagnes. L'exécution en a été assurée par l'Office du Niger.

Durant la première campagne (octobre 1957octobre 1958), avant tous r'ésultats du modèle, divers procédés ont été systématiquement essayés : dragages mécaniques, panneaux de fond, panneaux de surface; chacun de ces procédés agissant seul ou non.

En particulier, un dragage mécanique a été eflectué sur le seuil aval de Kassa (longueur de $200 \mathrm{~m}$ à la cote $-0,55 \mathrm{~m}$ sous l'étiage conven(ionnel). L'amélioration ne s'est pas maintenue jusqu'à la fin de la décrue; les dépôts se sont accumulés à l'aval de la zone draguée et le gain n'a été au total que de $0,10 \mathrm{~m}$.

Cette campagne a confirmé la validité de la méthode de correction par panneaux, dont les mises au point ont pu être effectuées grâce au modèle. Durant la seconde campagne (novembre 1958-mai 1959), les essais relatifs aux panneaux de fond et de surface ont été poursuivis, compte tenu des premiers résultats obtenus sur le modèle.

\section{Panneaux de fond.}

Pour la première campagne, les panneaux de fond étaient constitués par des écrans rectangulaires, dont l'étanchéité était assurée par des nattes, et qui étaient fixés sur le fond sableux par des rails battus. Ils formaient des ensembles de $50 \mathrm{~m}$ de longueur et de $1,40 \mathrm{~m}$ de hauteur.

Les trois seuils de la région de Kamani ont été équipés de panneaux de fond avec ou sans dragage préalable. Les dragages étaient surtout destinés à compenser la date tardive de mise en place de certains panneaux, l'époque d'action n'étant plus optimale (cote de $2 \mathrm{~m}$ à l'échelle de Koulikoro au lieu de $3 \mathrm{~m}$ ).

La cote critique des seuils a été abaissée de $50 \mathrm{~cm}$, mais certains panneaux se sont ensablés, comme le modèle l'avait prévu; d'autre part, Ies nattes, qui pourrissent assez rapidement, doivent être remplacées par des tôles, ce qui a été réalisé pour la seconde campagne.

Pour la seconde campagne, le modèle avait déjà permis de préciser certaines règles d'implantation des panneaux. Deux grands seuils (seuil moyen de Kamani et seuil amont de Kassa) furent équipés de panneaux de fond; l'angle des panneaux ne variait pas d'une facon continue
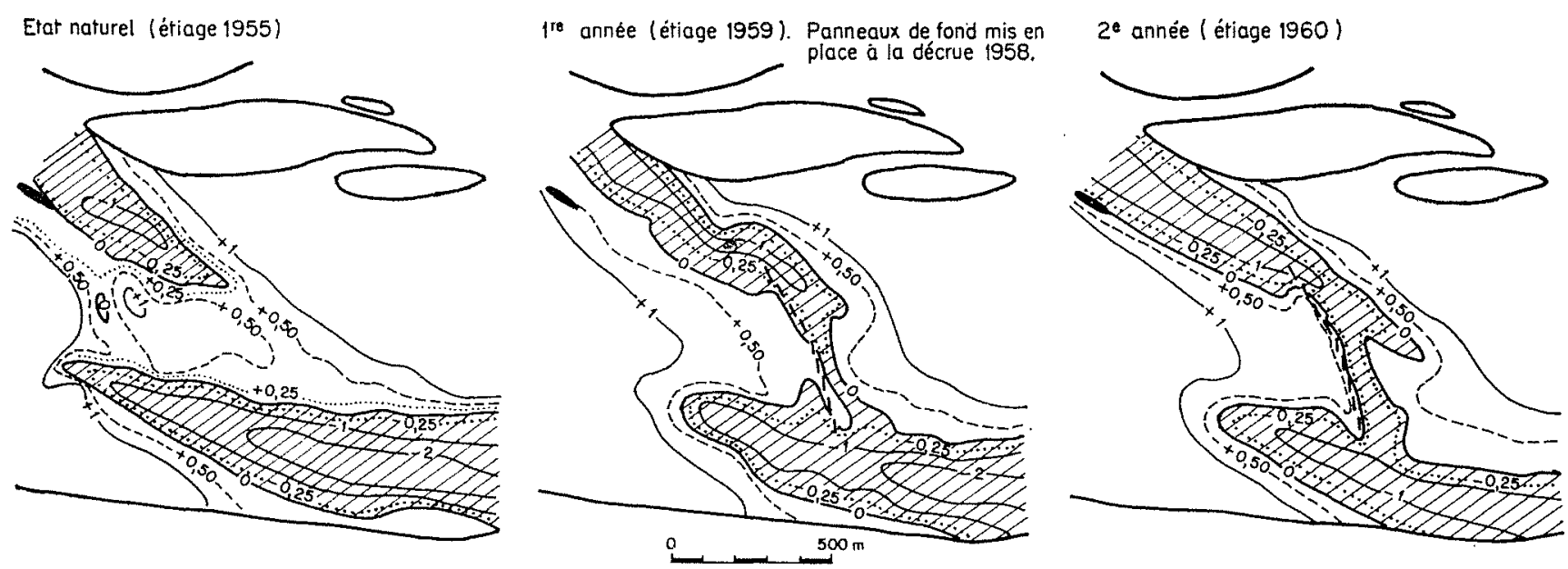

FIG. 13

Erolution d'un seuil aménagé de panneaux de fonds (région de Kassa). 
l'anont en aval; les panneaux étaient répartis sur deux axes distincls, l'un pour les panneaux amont, l'autre pour les panneaux aval.

Compte tenu des résultats des derniers essais effectués sur modèle, on sait que l'on peut obtenir un chenal mieux tracé et des approfondissements plus importants avec des panneaux dont les angles avec le courant varient d'une facon continue et qui sont également implantés sur une courbe continue. De plus, les approfondissements n'atteignent leur valeur limite qu'après jlusieurs décrues.

Les résultats furent les suivants (les creusements ćtant comptés par rapport à la cote maximale atteinte par les seuils avant tout travaux):

- seuil moyen de Kamani (fig. 11):

1958-59-Creusement de $1 \mathrm{~m}$ le long des sept panneaux amont et de $0,45 \mathrm{~m}$ le long des trois panneaux aval.

1959-60-Creusement d'au moins 0,75 m sur toute la longueur du chenal.

-. seuil amont de Kassa (fig. 13):

1958-59-Creusement de $0,50 \mathrm{~m}$ à $1 \mathrm{~m}$ le long des cinq panneaux amont el de $0,40 \mathrm{~m}$ le long des quatre panneaux aval.

1959-60-Creusement d'au moins $0,75 \mathrm{~m}$ sur toute la longueur du chenal.

Par exemple, en février 1960, pour une cote a l'áchelle de Koulikoro de $0,90 \mathrm{~m}$, alors que l'on avait moins de $0,50 \mathrm{~m}$ de tirant d'eau au senil aval de Kassa dans son élal naturel, on notait plus de $1,20 \mathrm{~m}$ de lirant d'eau au seuil amont aménagé de panneaux de fond en place depuis deux décrues.

\section{Panneaux de surface.}

A partir des résultats des essais préliminaires en canal, deux systèmes, formés chacun de 32 panneaux flottants, ont été construits pendant les deux campagnes. Chaque déflecteur mesure environ $2 \mathrm{~m}$; les ailes supportant ces déflecteurs ont respectivement $16 \mathrm{~m}$ de longueur pour les deux ailes amont, et $25 \mathrm{~m}$ de longueur pour les deux ailes aval. L'immersion maximale des panneaux est de $1 \mathrm{~m}$.

- Le seuil amont de Kassa a étć traité par ce procédé lors de la première campagne. Après vingt jours d'action (le débit du Niger variant de 400 à $200 \mathrm{~m}^{3} / \mathrm{s}$ ), un volume de $35000 \mathrm{~m}^{3}$ a été excavé; le chenal créé a une largeur de 50 à $100 \mathrm{~m}$ sur une longueur de $400 \mathrm{~m}$; le creusement minimal a été de $0,50 \mathrm{~m}$.

- Lors de la deuxieme campagne, un certain nombre de seuils ont été traités par les deux dispositifs. Les principaux résultats obtenus sont les suivants :

- Un creusement de $1,15 \mathrm{~m}$ a été obtenu après sept jours d'action sur un seuil court aux moyennes eaux de décrue;

- des creusements de 0,40 et $0,35 \mathrm{~m}$ ont étć obtenus, respectivement en 20 jours et 30 jours, sur des longueurs de 250 et $350 \mathrm{~m}$, sur deux seuils de longueur moyenne en fin de décrue (vitesses de courant très faibles, respectivement de 0,55 et $0,35 \mathrm{~m} / \mathrm{s}$ ).

- Enfin, en janvier et en février 1960, l'un de ces dispositifs a ćté utilisé pour mainlenir l'aceis du port de Koulikoro.

\section{CONCLUSION}

Le projel général de correction du bief Koulikoro-Ségou permet de gagner au moins $70 \mathrm{~cm}$ sur le tirant d'eau et de prolonger ainsi considérablement la période de navigabilité, surtout si l'on adapte la flotte à la technique du poussage. Alors que la navigation légère traditionnelle est interrompue en moyenne cinc mois par an, la navigation poussée allégée pourra être poursuivie sans interruption neuf années sur dix.

Il est intéressant de signaler ici que cette étude a pu être mence à bien grâce aux très nombreuses observations faites depuis plus de cinquante ans, et surtout à la liaison très étroite entre la mission sur place, dirigée par le Laboratoire, chargée des mesures et des travaux experimen- laux, et le Laboratoire chargé des études sur modèle.

L'évolution à long lerme du lit du Niger a été retrouvée sur modèle en reproduisant l'historique des débits du fleure; d'autre part, les nombreux sondases Jocaux, les mesures de charriage par traceurs radioactifs, et les mesures de vitesse effectuées par la mission sur place ont permis de contrôler dans son détail laptitude du modèle à reproduire tous les phénomènes intéressants.

La fidélité du modèle s'est trouvée ensuite confirmée, car il a été retrouvó ultérieurement dans la nature, soit le même ensablement des panneaux mis en place avant l'étude sur modèle 
lors de la première campagne, soit la même efficacité des ouvrages réalisés pendant la deuxième campagne, ouvrages qui avaient été mis au point sur le modèle d'ensemble du Niger après des essais d'orientation effectués en canal à grande échelle, avec ou sans distorsion, et sur différents fonds mobiles.
Cet ensemble de vérifications donne une garantie pour que l'efficacité à long terme des ouvrages projetés, telle qu'elle ressort des essais sur modèle, soit la même dans la nature, alors que, souvent dans ce domaine, le manque de données naturelles ne permet pas de conclure avec autant de certitude.

\section{I S C U S I O N}

Président: M. DE Rouvilue

M. Ropren insiste sur l'intérêt de tels aménagements. Le cas du Niger n'est pas isolé. Au cours d'études hydrographiques sur la Bénoué et le Chari, il a rencontré des conditions naturelles analogues. Il en est de même pour le secteur aval de l'Oubangui. Ces cours d'eau naturels exigent des débits limites de navigation enormes pour des tirants d'eau pas très élevés : $1600 \mathrm{~m}^{3} / \mathrm{s}$ poux $1,40 \mathrm{~m}$, par exemple, sur la Haute Bénoué. En outre, le faible tonnage transité : 40000 , 60000 ou 100000 tonnes par an, ne permet pas de réaliser des travaux de régularisation conteux. Les panneaux immergés apportent donc une solution très heureuse.

Sur la demande de M. le Président, M. Chabert indique :

$1^{\circ}$ que les panneaux de surface ne donnent qu'une amélioration temporaire (pendant une décrue par exemple), tandis que les panneaux de fond permettent de réaliser une correction permanente;

$2^{\circ}$ que, pour les travaux expérimentaux, les panneaux de fond étaient constitués d'éléments de $6 \mathrm{~m}$ de longueur et de $1,4 \mathrm{~m}$ de hauteur, placés les uns à côté des autres pour former un panneau de $50 \mathrm{~m}$ de longueur environ; chaque élément se composait d'un cadre métallique dont l'étanchéité était assurée, la première année par des nattes de fabrication locale, ensuite par des tôles ondulées soudées, et étaít fixé sur le fond au moyen de rails battus de 2 à $3 \mathrm{~m}$ dans le sable;

$3^{\circ}$ que pour des travaux en série, il y aurait peut-être intérêt à utiliser des panneaux formés d'un voile de béton:

4" que de nombreux cycles ont été reproduits sur modèle et laissent supposer que le chenal restera bien marqué pendant un grand nombre d'années après l'implantation des panneaux;

$5^{n}$ que, d'après les évaluations faites à Bamako, le coût des travaux de correction par panneaux de surface et de fond serait de 4 millions de nouveaux francs environ pour les $180 \mathrm{~km}$. L'évaluation du bénéfice certain résultant de la prolongation de la durée de navigabilité est assez délicate et sort de notre domaine.

M. le Président estime que le chemin de fer doit être bien matrais pour qu'on utilise la navigation.

M. Chabert mentionne qu'il est inexistant à partir de Koulikoro et que sa construction nécessiterait des investissements considérables.

M. le Président remarque que, d'autre part, le transport roulier est trés cher, car il n'y a que des pistes.
M. ZaOUi apporte deux compléments :

Le premier concerne des panneaux du même genre qui ont été placés en Tunisie en 1953, pour dessabler un émissaire de la rivière de la Medjerda causant des crues à l'aval : au bout d'une première séric de crues, la base a été dégravée à environ $50 \%$, ce qui montro bien l'efficacité d'un tel système.

Le deuxième, un peu anecdotique, rappelle que lc Professeur Potapov aurait pris un brevet de son invention, mais, n'ayant pas payé les redevances, il n'aurait pu en profiter.

M. Chabert signale que par un procédé analogue, il a étudié au Laboratoire les moyens propres à éviter l'ensablement de la prise d'eau de Markala qui contrôle l'irrigation de l'Office du Niger. D'autre part, le Laboratoire avait également mis au point sur modèle des dispositifs de panneaux de surface pour empecher l'engravement d'anciennes prises d'eau d'aménagements hydroélectriques sur la Durance (la Saulce, par exemple); mais des solutions plus classiques avaient été préférées à l'époque.

M. le Président précise que e'est une méthode susceptible d'être généralisée dans les pays très secs à pluriosité discontinue.

M. Gridel, appuyé par M. Chabert, signale, pour répondre à la remarque anecdotique de $M$. $Z_{A}$ ou, que, suivant l'implantation mise au point sur modèle, les panneaux de fond forment un système discontinu, que leur écartement dépend de la vitesse du courant, et que leur inclinaison varie d'amont vers l'aval de facon $\dot{a}$ obtenir une efficacité maximale, compte tenu de la courbure que présentent les courants lor's de la décrue. Le dispositif diffère donc totalement de ceux préconisés par Potapov, puisque le Laboratoire a pu valablement breveter son dispositif.

M. le Président ajoute qu'il faut faire des économies de panneaux, parce que les dépenses peuvent devenir importantes; en dehors des zones marines, cette discontinuité ne nuit pas à la conservation des panneaux.

M. Chamboredon veut attirer lattention sur les données qui paraissent conditionner, et par suite limiter, Ia validité pratique de la méthode de correction au moyen de panneaux déviateurs dont a parlé M. Chabert.

$1^{\circ}$ Ainsi que $M$. le Président vient de le signaler, une telle méthode, en raison du mouillage relativement faible susceptible d'être obtenu, ne saurait être envisagée que lorsque la voie d'eau à améliorer constitue l'unique moyen de transport, sans route ni voie fercée concurrente à grand rendement desservant les mêmes relations; 
$2^{\circ}$ La méthode par panneaux déviateurs est intéressante dans les régions où l'absence de tout matériau rochcux interdit pratiquement le recours aux ouvrages classiques en enrochements (épis et digues);

$3^{\circ}$ L'application en est d'autre part favorisée par un régime hydraulique régulier du fleuve ou de la rivière, sans variation brutale et fréquente des vitesses, ce qui doit permettre à la fois une bonne tenue des panneaux et leur action efficace.

Ces conditions se rencontrent, pense-t-il, sur les fleuves africains ainsi que sur les fleuves russes, où la méthode des panneaux déviateurs a pris naissance.

Par contre, l'application d'un tel procédé en Franee paraît devoir rester limitée à certains problèmes très particuliers, où des circonstances exceptionnelles peuvent recommander l'emploi de panneaux déviateurs.

M. Chazert répond qu'en effet, dans la zone fu Niger à aménager, il est très difficile de trouver des enrochements, et que, d'autre part, pendant les crues du Niger les vitesses n'augnentent pratiquement pas (elles sont toujour's de l'ordre de $1 \mathrm{~m} / \mathrm{s}$ au maximum), car le champ d'inondation peut s'étendre largement.

M. le président pense, comme M. Chamboredon a voulu le laisser entendre, que si l'on avait voulu mettre de tels panneaux dans le Rhône, ils n'auraient pas tenu.

M. Chabent indique par contre que sur la Loire (immédiatement en amont de la zone maritime), dont le lit sableux est un peu analogue à celui de certaines rivières africaines, il est prévu d'effectuer des essais de panneaux de surface pour faciliter la navigation sur les seuils en période d'étiage. Un dispositif a déjà èté réalisé cette année; mais depuis, les conditions hydrologiques n'ont pas permis d'éprouver le système.

M. Ie Président clôt le débat en remerciant encore M. Chabert d'avoir bien voulu exposer ces problèmes intéressants.

\section{NOTRE FRONTISPICE}

\section{(C). page 766)}

\section{HeWI ROINCARE (1854-1912)}

La trop courte carrière d'Henvi Poincarć a été singulièrenent brillante. A l'âge de trente ans scs premiers travaux analytiques sur les groupes fuchsiens et kleinéens le mettaient déjà hors de sur

Si la Mécanique Céleste (problème des $n$ corps) fut son premier champ d'applications, il passe rapidement de là à la physique mathématiçue. Il résoud notamment le problème des marees, montrant que sa solution générale se ramène à une équation ou à un système de deux équations intégrales de Fredholm. La solution particulière “cylindrique » dite "onde de Sverdrup» est déjà explicitée dans son étude.

Il ne traite pas senlement de la physique mathématique en aualyste. Sur les sujets les plus variés : élasticité, hydrodynamique, theorie de la chaleur, thermodynamique, capillarite, optique, électricité, il se révèle comme un Maitre, excellant à mettre à nu les mécanismes analytiques qui, sous des manteaux divers, se retrouvent partout en physique et dont son esprit critique aime à signaler les difficultés et les contradictions. Moins soucieux de resoudre définitivement des problèmes que de se poser a leur sujet de multiples questions ou de mettre en cenvre des procédés

Les hydrauliciens lisent encore avec émerveillement le cours de "Mécanique des fluides" qu'il professa en 1891 à l'Université le Paris. Sans résondre complètement le paradoxe de d'Alembert, i1 y développe les recherches d'Helmholtz, montrant conment la connaissance des tourbillons fournit celle des vitesses, se ranenant à l'équation de Poisson par une formule ingénieuse devenue classique, notamment en électronagnétisme :

$$
\operatorname{rot} \operatorname{rot} \vec{V}=\operatorname{grad} \operatorname{div} \vec{V}-\overrightarrow{\Delta V}
$$
Sa thécrie des nappes de discontinuité à l'arrière l'un obstacle anulonce ct, dans une certaine mesure confirme, la configuration plus précise, fondée sur la "couche limite
1920 par Prandtl et l'école de Göttingen.

Les mathématiques et la physicue le mènent à la philosophie des sciences où ses nombreux onvrages ont fait connaitre son nom à un public étendu. Ses développements sur la géomćtrie de situation (Analysis gitus) où il apmait comme un précursentr des topologistes. sa critique des axiomes de la mécanic, ue classique, ses réflexions sur les quanta (1911) ont ćté largement diffusés. II n'a pas it proprement parler de philosophie en ce sens gu'il n'at pas cherché à édifier un systène mais plutôt à critiquer et it méditer sous forme de "pensées». Ainsi partage-t-il avec Pascal cette gloire singulière d'être revendiqué comme un des leurs par les écoles les plus opposées: dogmationes comme sceptiques, classigues et réplutionnires.

Un mot revient souvent dans ses écrits, oi certalins ont vu lat caractéristique de sa pensée: la commodité, c'est à-dire la simplicité visant à l'efficacité. Ce souci majeur, $r_{2}$ ui fut le sien, nous rappelle que le mathematicien, le physicien, le philosophe, forme à l'Ecole Polytechnique d'où il était sorti dans le Corps des Mines, fut malgré tout d'abord un ingénieur.
HENRI POINCARE (1854-1912)

Though short, Heuri Poincarćs carcer rous outstandingly brilliant, His early analytical asork on Fuchsian and Kleinian groups had alveady confirmed his exceptional ability at the age of thirty.

From celestial mechanics (problem of the $\mathrm{n}$ bodies), sulich was his original ficld of research, he soon transfored his interest to mathematical physics. His most important achievement in this ficld was his solution of the tide problem, in which he showed that his general solution reduces to a single equation or to a system of two Fredholm integral equations. An explicit statement of the "cylissdrical" particular solution knoz'n as the "Swerdrup wave" is alveady found in this study.

Honri Poincarés approach to problems of mathematical physics was by no means alatays an analytical one. He was also a leading authority on such varied subjects as elasticity, hydrodynamics, theory of heat, capillarity, optics and electricity, in which he passessed the singular gift of being able to expase those analytical mechanisms which occur in various disgnised forms in all branches of physics, the difficultics and contradictions of a.hich his critical mind cujoyed bringing to light. He zeas less concerned with finding definite solutions to problems than with asking himsclf numerous questions about them and drveloping new' methods ("Balayage" method).

Hi scrics of lectures on Fluid Mechanics at the University of Paris in 1891 is still greatly admired by hydranlics cugincers tolay. Without complctely solving d'Alembert's paradox, Ite ne'crtheless developed Helmholtz's findings, showing how the aclocitics can be detcrmincd if the eddies are know, and arriving at Poisson's equation by means of the follozing ingentous formula:

$$
\text { rot vot } \vec{V}=\text { grad div } \vec{V}-\overrightarrow{\Delta V}
$$

aithich is now a classical relation, especially in clectromannetism. His theory of wakes behind an obstacle heralded -and to some crtcnt even confirmed-the more precise "boundary layer" concept first stated by Prandtl and the Göttingen school in 1920. After mathematics and physics, Henri Poincaré eventually turned his atcotion to scientific philosophy in which he became thided his attention to scientife philosophy, in which he became quidely the ucometry of place (Aualysis situs) in which he arthears on the geometry of place (Analysis situs), th which he appears as a forernnuer of the modern topologists, his criticism of the arroms of classical mechanics, and his thonghts on quanta 1911) were widely circulated. He did not specifically discuss philosophy, inasmuch as he made no attempt to actually construct system; his aim was rather to criticize and meditate in the form of "Thoughts". He thus shares with Pascal the distincdogmatists and sceptics, or classics and revolutionaries. Many see the characteristics feature of Poincaré's trend of thought in a word he often repeats in his works, the word "conght in a word he often "repeats in, his works, the work

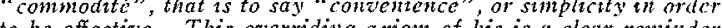
to be effective. This overriding axiom of his is a clear reminder of the fact that Poinare the mather hhilosopher, monlded by the Ecole Polylechnilne, from anch he yraduated with Honours, was nevertheless crifiner. 